\title{
Intensity Variations of the Soft X-ray Background: the Boundary Structure of the Local Hot Bubble at Low Galactic Latitudes
}

S. Park ${ }^{1}$, J.P. Finley ${ }^{1}$, and S.L. Snowden ${ }^{2,3}$

${ }^{1}$ Department of Physics, Purdue University, 1396 Physics Building, West Lafayette, IN. 47907, USA

2 NASA/Goddard Space Flight Center, Code 662, Greenbelt, MD. 20771, USA

${ }^{3}$ Universities Space Research Association, USA

Abstract. 42 ROSAT PSPC pointed observations in the Galactic plane $\left(l \sim 4^{\circ}-\right.$ $26^{\circ}$ ) are mosaicked in order to study the spatial structure of the X-ray emitting gas in the Local Hot Bubble (LHB). Degree scale X-ray intensity variations are detected at the $\pm 10 \%$ level in the $\frac{1}{4} \mathrm{keV}$ band, which imply a likely influence from a clumpy boundary shell of the LHB in the observed $\frac{1}{4} \mathrm{keV}$ band X-ray background. The possible origins of such a clumpy boundary structure of the LHB are discussed.

\section{Introduction}

In the Galactic plane, the $\frac{1}{4} \mathrm{keV}$ band soft $\mathrm{X}$-ray diffuse background (SXRB) is expected to originate within the LHB due to the substantial absorption cross-section of the ISM (e.g., $\tau \sim 1$ at $\sim 30 \mathrm{pc}$, assuming $\mathrm{n}(\mathrm{H}) \sim 1 \mathrm{~cm}^{-3}$ ). This "isolation" from the contribution of any flux of a more distant Galactic origin allows for the study of the detailed structure of the LHB by searching for the $\frac{1}{4} \mathrm{keV}$ band $\mathrm{X}$-ray intensity variations at various angular scales. Here we report detection of degree scale variations of the $\frac{1}{4} \mathrm{keV}$ band SXRB in the Galactic plane $\left(l \sim 4^{\circ}-26^{\circ}\right)$, which implies an influence by a shell-like boundary structure of the LHB on the observed $\frac{1}{4} \mathrm{keV}$ band SXRB.

\section{Data}

An R1L and R2 band (Snowden et al. 1994) mosaic of 42 ROSAT PSPC pointed observations are used in this study. All identified non-cosmic backgrounds ( $\sim 22 \%$ of the total counts) are modeled and subtracted from the data as described in Snowden et al. (1994). The detected point sources and possible enhancements by SNRs and X-ray binaries are removed and the relative offsets between overlapping pointings are corrected $(\sim 9 \%)$. The final mosaic (Figure 1) covers $\sim 60$ degree $^{2}\left(l \sim 4^{\circ}-26^{\circ}, b \sim-3^{\circ}-+2^{\circ}\right)$ with an average exposure of $\sim 8 \mathrm{ks}$. With a $10^{\prime}$ binning, an average of $6 \%$ statistics per pixel is achieved in the R1L+R2 band (Park, Finley, \& Snowden 1997 for a detailed description of the data). 


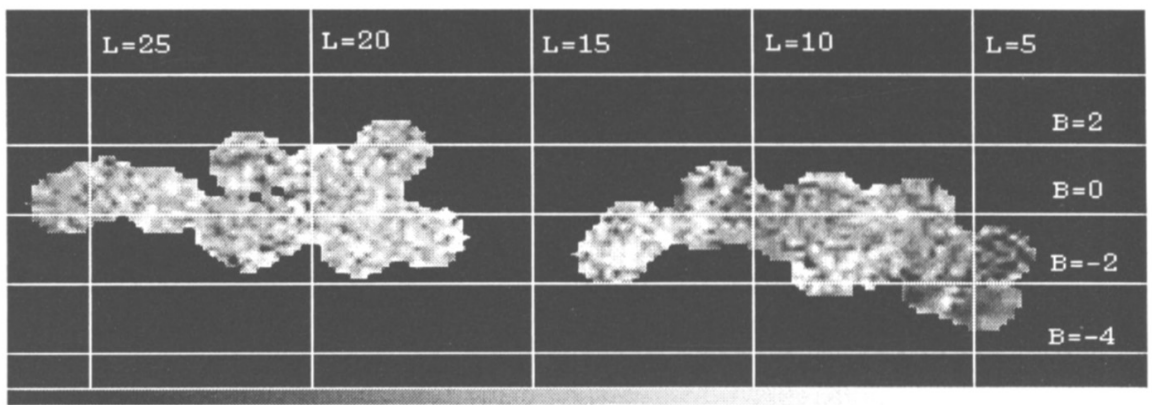

Fig. 1. The $\frac{1}{4} \mathrm{keV}$ band (R1L+R2) mosaic of the 42 ROSAT PSPC pointings in the Galactic plane. The pixel size is $10^{\prime}$ and the data have been smoothed. The gray-scale ranges $0-800 \times 10^{-6}$ counts s${ }^{-1} \operatorname{arcmin}^{-2}$.

\section{Analysis and Results}

In order to search for X-ray intensity variations along the Galacticplane, the data are first integrated across the plane in Galactic latitude, $b$, to create $10^{\prime}$ columns, typically within $\pm 2^{\circ}$ from the plane. With this integrated $1-\mathrm{D}$ binning, the $\mathrm{R} 1 \mathrm{~L}+\mathrm{R} 2$ band intensity variation along the plane is displayed in Figure 2a. The average flux is $\sim 400 \times 10^{-6}$ counts $s^{-1} \operatorname{arcmin}^{-2}$. The $\mathrm{X}$-ray intensity is spatially variable (reduced $\chi^{2}>5$ about the mean) at the $\pm 10 \%$ level. A spatial Fourier transform reveals degree scale variations with an $\sim 5.5^{\circ}$ scale being the most prominent in all bands (Figure $2 \mathrm{~b}$ ). Sub-degree scale variations are investigated with a $2-\mathrm{D}$ autocorrelation function (ACF). The ACF at angular scales $\leq 3^{\circ}$ is displayed in Figure 3. The ACF in both the R1L and R2 bands indicates little correlation (formal errors include zero) at angular scales of $\leq 3^{\circ}$. The difference of the ACF between the three bands (R1L, R2, and R1L+R2) is not significant and lies within the statistical uncertainties. The average hardness ratio (1.35) of the mosaic implies a plasma temperature of $\sim 10^{6.1} \mathrm{~K}$ with no absorption which is consistent with that of Snowden et al. (1997) for emission from the LHB.

\section{Discussion}

Possible origins of the detected variations are discussed below.

\subsection{Magnetic Rayleigh-Taylor (R-T) Instability}

A SNR in the adiabatic phase is R-T unstable. Assuming an $\sim 10^{5}$ year old blast wave in a pre-existing local cavity $\left(n \sim 0.004 \mathrm{~cm}^{-3}\right)$ "reheated" by a $\mathrm{SN}$ explosion, the critical wavelength of the magnetic R-T instability, $\lambda$, can be estimated (Table 1) with a typical Galactic midplane magnetic field $\mathrm{B}=$ 

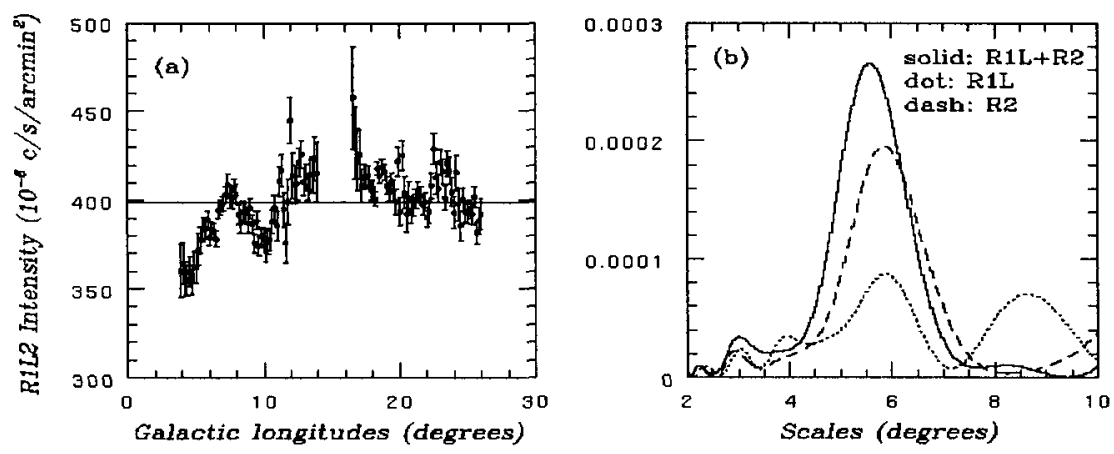

Fig. 2. (a) The $\frac{1}{4} \mathrm{keV}$ band intensity along the Galactic plane. Each point presents a $10^{\prime}$ column integrated across the plane. The horizontal line is the mean. (b) Spatial Fourier transform of the R1L, R2, and R1L+R2 band X-ray intensities .
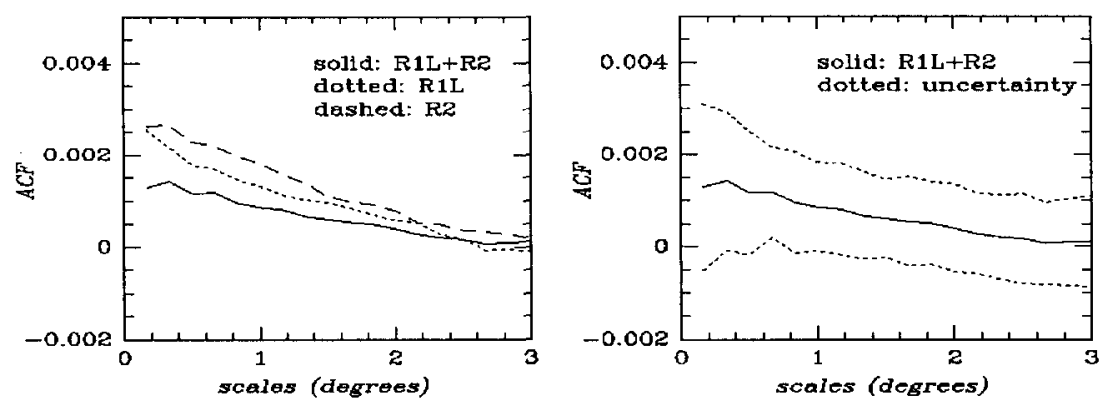

Fig. 3. The ACF of the $\frac{1}{4} \mathrm{keV}$ band $\mathrm{X}$-ray intensity for angular scales $\leq 3^{\circ}$. The left panel shows the ACF for the R1L, R2, and R1L2 bands. The right panel shows the R1L2 band ACF and its formal uncertainty.

$5 \mu \mathrm{G}$, a plasma temperature of the LHB $\mathrm{T} \sim 10^{6.1} \mathrm{~K}$, and a stored thermal energy $\mathrm{E}_{T H}=1-3 \times 10^{50} \mathrm{ergs}$ (see Park, Finley, \& Snowden [1997] for the detailed calculation). The range of $\lambda$ is remarkably consistent with the detected $\sim 5.5^{\circ}$ scale variation given the inaccuracies embedded in the simple spherical blast wave model. With this model the detected $\pm 10 \%$ variation at $\sim 5.5^{\circ}$ scale can be attributed to the path-length variation of the wave-like boundary structure due to the magnetic $\mathrm{R}-\mathrm{T}$ instability.

\subsection{Inhomogeneous ISM}

If the blast wave of the LHB is in the radiative phase $\left(\tau \gtrless 10^{6} \mathrm{yr}\right.$ ), the boundary shell can become clumpy due to inhomogeneities in the ISM. For 
Table 1. Modeled $\lambda$ of a magnetic R-T instability at the boundary of the LHB

\begin{tabular}{cccc}
\hline$R_{L H B}(\mathrm{pc}) E_{T H}$ & $\left(10^{50} \mathrm{erg}\right)$ & $\lambda(\mathrm{pc})$ & Observable angular size $\left(^{\circ}\right)$ \\
\hline \multirow{2}{*}{50} & 1.0 & 1.9 & 2.2 \\
& 2.0 & 4.1 & 4.7 \\
\multirow{3}{*}{75} & 3.0 & 5.8 & 6.6 \\
& 1.0 & 0.8 & 0.9 \\
& 2.0 & 1.7 & 2.0 \\
& 3.0 & 2.5 & 2.9 \\
\hline
\end{tabular}

example, absorption by typical interstellar cloudlets (angular size $2-5 \mathrm{pc}$, internal density $1-3 \mathrm{~cm}^{-3}$, Heiles 1967) are reasonable to produce detected $\$ 5.5^{\circ}$ scale variation at $\pm 10 \%$ level.

\subsection{Emission Variations at the Boundary Layer}

The emission variation at the LHB boundary such as electron density fluctuation (Phillips \& Clegg 1992) may also produce the observed intensity variations.

\subsection{The Local Fluff (LF) and Embedded Clouds}

Due to the low $N_{\mathrm{H}}\left(\$ 3 \times 10^{18} \mathrm{~cm}^{-2}\right)$, the LF is unlikely to be the source of the $\pm 10 \%$ variation (required $N_{\mathrm{H}} \sim 10^{19} \mathrm{~cm}^{-2}$ ). Observations of local interstellar clouds with $N_{\mathrm{H}} \gtrless 10^{19} \mathrm{~cm}^{-2}$ inside of the LHB (e.g., Kerp, Herbstmeier, \& Mebold 1993) may indicate that the detected variations can be due to absorption by embedded clouds.

\section{Conclusions}

Degree scale intensity variations (at $\pm 10 \%$ level) in the $\frac{1}{4} \mathrm{keV}$ SXRB are detected in the Galactic plane. The origin of these small-scale variations is most likely the presence of R-T instability and/or clumpy cooler ISM at the boundary of the LHB. Variations due to absorption by embedded clouds cannot, however, be ruled out.

\section{References}

Heiles, C. 1967, ApJS, 15, 97

Kerp, J., Herbstmeier, U., \& Mebold, U. 1993, A\&A, 268L, L21

Park, S., Finley, J. P., \& Snowden, S. L. 1997, ApJ, 491, in press

Phillips, J. A. \& Clegg, A. W. 1992, Nature, 360, 137

Snowden, S. L. et al. 1994, ApJ, 424,714

Snowden, S. L. et al. 1997, ApJ, submitted 